\title{
Designing CMC Tools for Separated Families
}

Negar Khojasteh

Cornell University

Ithaca, NY 14850, USA

nk586@cornell.edu
Permission to make digital or hard copies of all or part of this work for personal or classroom use is granted without fee provided that copies are not made or

the full citation on the first page. Copyrights for components of this work own and others than the author(s) must be honored. Abstracting with credit is permitted. To copy otherwise, or republish, to post on servers or to redistribute to lists, requires prior specific permission and/or a fee. Request permissions from

Permissions@acm.org.

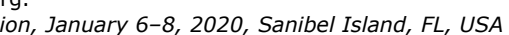

(C) 2020 Copyright is held by the owner/author(s). Publication rights licensed to

ACM.

ACM 978-1-4503-6767-7/20/01\$15.00

https://doi.org/10.1145/3323994.3372140

\begin{abstract}
Today, millions of people live in a country other than their home country, for example as international students, or as immigrants. Computer-mediated communication (CMC) tools such as video conferencing applications, help people stay in touch with their families and friends back home. Despite their use as a means of remote communication, CMC tools are very limited in affording users' needs that are beyond conversation, such as cooperative activities or enjoying a shared experience. For my dissertation, I am proposing the following aims. First, I will investigate the shortcomings of CMC tools in addressing people needs in remote communication. Secondly, based on this understanding, I will propose design implications that will address these shortcomings.
\end{abstract}

\section{Author Keywords}

Computer-mediated communication; separated families; virtual reality, MRP

\section{CCS Concepts}

CCS $\rightarrow$ Human-centered computing $\rightarrow$ Collaborative and social computing $\rightarrow$ Empirical studies in collaborative and social computing 

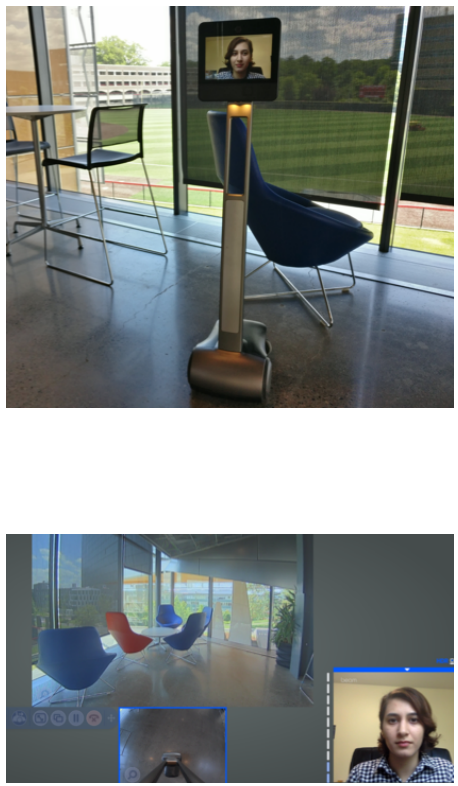

Figure 1: (top) MRP used in the both project 1 and 2, Beam by suitable technologies. Screen shows remote user. (bottom) Participant's view of the remote location via MRP software.

\section{Introduction}

People who live far from their families use CMC tools as their only option to overcome the physical distance impeding direct communication with their loved ones. Features such as video calling have been particularly useful in mimicking an actual face to face conversation. However, face to face communication is not the only interaction distant family members miss. By living apart, one misses the opportunity to participate in activities and shared experiences with physically absent family members and friends.

The main goal of my doctoral research is to find strategies for leveraging technology to provide users with the ability to have shared experiences and perform cooperative activities with their family members. Using qualitative studies to investigate users' expectations and needs, I plan to design features for current CMC tools, as well as exploring more recent technologies such as Virtual Reality (VR) as potential solutions for fulfilling the need for togetherness in physically separated families.

From my previous projects, I have had the opportunity to use mobile robotic telepresence (MRP) systems and virtual reality environments (VR) in the context of remote team collaboration. MRP provides users with an audio-video channel, stationed on a robotic base, which can then be controlled by users remotely (figure 1). With MRP, the remote user (also called the pilot) can communicate with the people who are in the remote location as well as controlling and navigating the MRP in the space where it is physically located. Previous work has shown the benefits of MRPs such as the feeling of sharing a home and enjoyment of companionship [4] for couples in long distance relationships. Considering these benefits for couples, MRPs could be beneficial for individuals who live far from their families as well. In VR, users can create their own avatar and communicate with others in a shared virtual space. Both of these provide affordances beyond the ones offered by the traditional CMC tools. Mobility and physical embodiment of MRP, and the fully immersed shared environment, that is offered by VR, are features that can enhance sense of togetherness and connection for users, who cannot interact directly, by allowing them to engage in shared activities.

In the following sections, I will explain the studies I have done so far as well as my aims for subsequent research projects. I will conclude with a brief note on the expected contribution and my objective for attending this doctoral consortium.

\section{Project Overview}

Project 1: Mobile robotic telepresence (MRP) and Teamwork (in preparation for review)

In a series of three lab studies (120 sessions total), I worked on three design solutions for helping remote users with the task of controlling a telepresence robot. Previous work has shown that controlling the robot can be challenging (e.g., [3]). In these lab studies, I tested three assisted driving options: an autonomous personfollowing MRP, a fully autonomous goal-based MRP, and shared control of MRP by two users. Findings of these studies suggest several considerations that should be taken into account in designing autonomous MRPs. For example, future autonomous MRPs should always provide users with a manual option, because we have found that people preferably use the manual position adjustments in signaling their attention to users who are collocated with the MRP. 
Project 2: MRP in Campus Setting (completed) I did a qualitative study on use of MRPs in campus setting with the goal of exploring challenges students face when they use MRPs for everyday tasks [2]. I observed participants using an MRP for several sessions and interviewed them for learning about their experiences. Analysis of those interviews revealed that students have self-presentation concerns when they drive the MRP, and find it difficult to approach people with their face on the robot. I then proposed design solutions that could help students overcome these challenges and have a better experience when using an MRP.

Project 3: Iongitudinal Virtual Reality Study (under review)

I designed and conducted a longitudinal VR study to explore how emotions are interpreted in VR. Most studies in social VR are one-time in the laboratory or field studies. Few studies have looked at changes in users' social behavior over time (e.g., [1]). I recruited 20 participants to collaborate on a game-like task in weekly laboratory VR sessions. Over the course of five weeks, participants filled out an online survey after each session, and all participants were interviewed after the last session. We found that participants mostly rely on tone of voice for expressing and interpreting other users' emotions. Consistently, the participants rarely used the visual emotion features available on avatars. We also found that, over time, user report higher sense of presence and immersion in the virtual environment.
Project 4: Interview study with international students (ongoing)

Currently, I am in the middle stages of an interview study as the initial step towards designing CMC tools for international students and, more broadly, separated families. I have been recruiting international students and I asking them about their experiences of studying abroad and their practices around use of CMC tools for communication with family and friends. In the interview, I inquire about the activities they are interested in doing with their families and experiences they would like to share with them over distance. I plan to use the findings of this study as the basis for the next projects described below.

Project 5: Designing CMC tools for separated families (planned)

The information gathered in Project 4 will provide the initial insights into designing features for current CMC tools. I also plan to take advantage of the findings of Project 1-3 regarding remote team collaboration to further enhance the effectiveness of modified CMC tools for interpersonal communication. The ultimate goal is to improve users' sense of social presence and togetherness when they use CMC tools to interact. An essential part of this project will involve having users test the implemented designs and obtaining their feedback for further improvements in an iterative process.

\section{Expected Contribution}

My dissertation work will contribute to the field of $\mathrm{CMC}_{\text {, }}$ virtual reality, and interpersonal communication. The ongoing interview studies will aid in better

understanding of separated families. The findings of these studies will shed light on the challenges people 
face in sharing their experiences and activities over distance. I will use the qualitative results of the interviews to address the needs of this population regarding social connection with their families by improving the current $\mathrm{CMC}$ tools and designing new applications. Additionally, I will investigate the potential benefits of recent technologies such as MRP systems and VR environments for this population.

\section{Objective for GROUP Doctoral Consortium}

I believe that attending the doctoral consortium at GROUP 2020 will be greatly valuable for my PhD experience. Given that my research is focused on CMC and interpersonal communication, I am confident that my work is relevant to the attendees of this conference and I think the comments and feedbacks from senior researchers and PhD students at the consortium will help me with improving my research plan. By attending this doctoral consortium, I mainly look for insights and suggestions on potential ideas and studies that I can incorporate in my research plan. Additionally, as a member of the research community who has collaborated on several projects and reviewed journal and conference papers in the past three years, I look forward and am excited to discuss other students' projects with them. I believe my cultural background and experiences as a woman from Middle East, as well as being an international student, has equipped me with a unique perspective to participate in GROUP Doctoral Consortium.

\section{Acknowledgements}

I thank my great advisors Susan R. Fussell, Malte F. Jung and Andrea Stevenson Won for guiding and supporting me. This work was supported in part by National Science Foundation award IIS-1563705.

\section{References}

1. Jeremy N. Bailenson, and Nick Yee. 2006. A longitudinal study of task performance, head movements, subjective report, simulator sickness, and transformed social interaction in collaborative virtual environments. Presence: Teleoperators and Virtual Environments, 15(6), 699-716.

2. Negar Khojasteh, Cathy Liu, and Susan R. Fussell. 2019. Understanding Undergraduate Students' Experiences of Telepresence Robots on Campus. In Companion of the 2019 ACM Conference on Computer Supported Cooperative Work and Social Computing (CSCW '19). ACM, New York, NY, USA. DOI: https://doi.org/10.1145/3311957.3359450

3. Min Kyung Lee and Leila Takayama. 2011. "Now, I have a body": uses and social norms for mobile remote presence in the workplace. In Proceedings of the SIGCHI Conference on Human Factors in Computing Systems (CHI '11). ACM, New York, NY, USA, 33-42. DOI: https://doi.org/10.1145/1978942.1978950

4. Lillian Yang and Carman Neustaedter. 2018. Our House: Living Long Distance with a Telepresence Robot. Proc. ACM Hum.-Comput. Interact. 2, CSCW, Article 190 (November 2018), 18 pages. DOI: https://doi.org/10.1145/3274459. 